\title{
Just we begin the new era of Korean Journal of Transplantation
}

\author{
Ik Jin Yun
}

Editor-in-Chief, Korean Journal of Transplantation

This year is the 50th anniversary of the Korean Society for Transplantation (KST). During the past 50 years, many changes have occurred in the clinical and research fields of organ transplantation both globally and in Korea. Especially for the KST, clinical achievements just like those in the field of living liver transplantation and the developments of basic research, such as stem cell research and xenotransplantation experiments, are outstanding. Korea has become one of the leading countries in the field of transplantation both in the clinical and research fields.

However, the role of the Korean Journal of Transplantation (KJT), the official journal of the KST, with 32 years of publication, does not sufficiently reflect the developments and achievements in Korean transplantation. At the beginning, the KJT was the only and valuable representative journal of the KST. However, against the expansion of the KST and medical field of transplantation, the role and value of the KJT is decreasing. Thus, we have changed all the submission and publication systems and required authors to use only English for their articles for publication. We are just becoming a responsible international medical journal.

Now, during the time of these changes, we promise several things to authors and readers. One, we will comply with our publication purposes and aims more thoroughly. In the KST and Asian Society of Transplantation, problems and views differ from those of the Western society. Ethical and legislative problems are more serious, and the situation of organ donation and level of research are very different from those in other regions. Thus, we will make more efforts to reflect the characteristics of our regional academy. Two, nowadays, the commercialism of the scientific journals has increased to the extent that it has increased the financial burden on authors if one seeking to publish an article in a good journal. We will work to develop a good journal at the SCI (Science Citation Index) level without imposing undue financial burden to authors. Three, we intend to develop a publication system that reflects well the changing times. The most important purpose of publication in a scientific journal is easier communication; therefore, we promise to ensure easy accessibility and convenience for authors and readers.

We will start with small changes. However, we are confident that these changes will be the achievements that members of the KST and world society of transplantation can be proud of.

\section{ORCID}

Ik Jin Yun https://orcid.org/0000-0003-4013-6714 